\title{
RSV BRONCHIOLITIS: VALIDATION OF THE DIAGNOSIS IN HOSPITALIZATIONS
}

Mónica López-Lacort', Cintia Muñoz-Quiles', Alejandro Orrico-Sánchez', Javier Díez-Domingo'

${ }^{\prime}$ Vaccine Research Department, FISABIO-Public Health, Valencia, Spain

To validate the reliability of the bronchiolitis cases due to Respiratory Syncytial Virus (RSV) recorded in the Minimum Basic Data Set (MBDS), by calculating the positive predictive value (PPV) using the Valencia Microbiological Network (RedMiVa) as the reference standard.

\section{Methods}

A retrospective population-based study using health databases of Valencia Region.

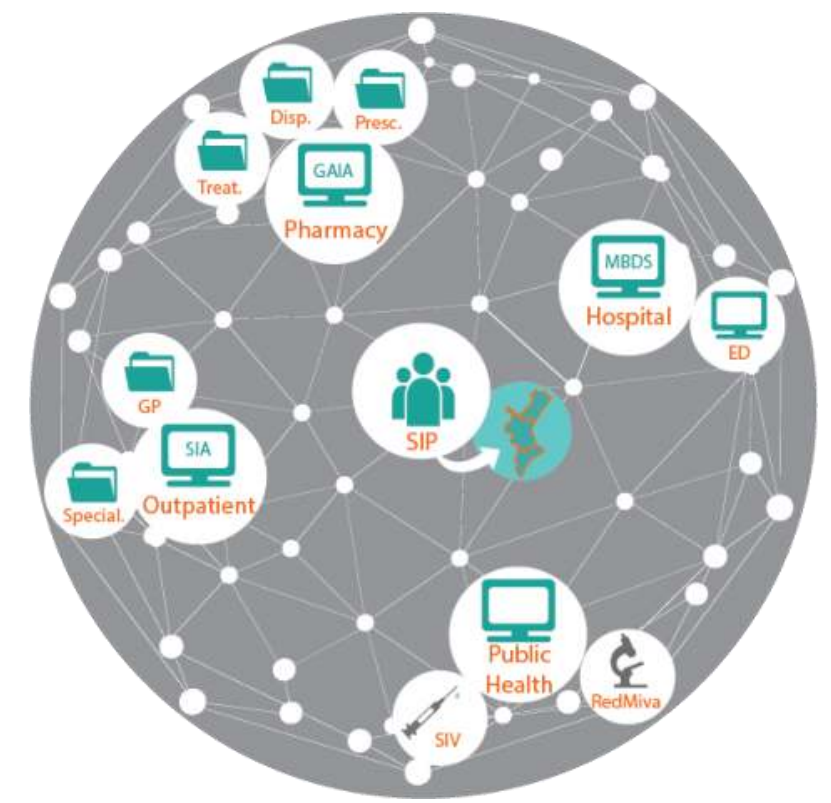

Health databases of Valencia Region

Population: Children under 2 years of age born between 2008 and 2012 with an admission for RSV bronchiolitis. Period: 2008-2012.

Case definition: First admission for bronchiolitis (ICD-9 466.11) in first diagnostic position. (Mc Connochie definition)

PPV estimation: $20 \%$ of the hospitalizations do not have an associated test result, this missing information was estimated by multiple imputation. The imputation and PPV were assessed using a Bayesian model adjusted by age, year of admission and hospital as a random effect.

\section{Results}

Of the $\mathbf{3 7 0 7}$ RSV bronchiolitis hospitalizations, $96.6 \%$ occurred in children under one year of age. Among them, 20.3\% ( $n=753$ ) had no associated laboratory test, $4.3 \%$ ( $n=160$ ) had negative test result and $75.3 \%$ ( $n=$ 2790) had positive test result. According to the imputation model, the estimated PPV was:

$$
93.7 \% \mathrm{Cl}(95 \%)(92.7-94.7)
$$

\begin{tabular}{cccc}
\hline & \multicolumn{3}{c}{ RedMIVA (Gold standard) } \\
\hline MBDS & + TEST & - TEST & MISSING \\
466.11 & 2790 & 160 & 753 \\
\hline
\end{tabular}

Other strategies

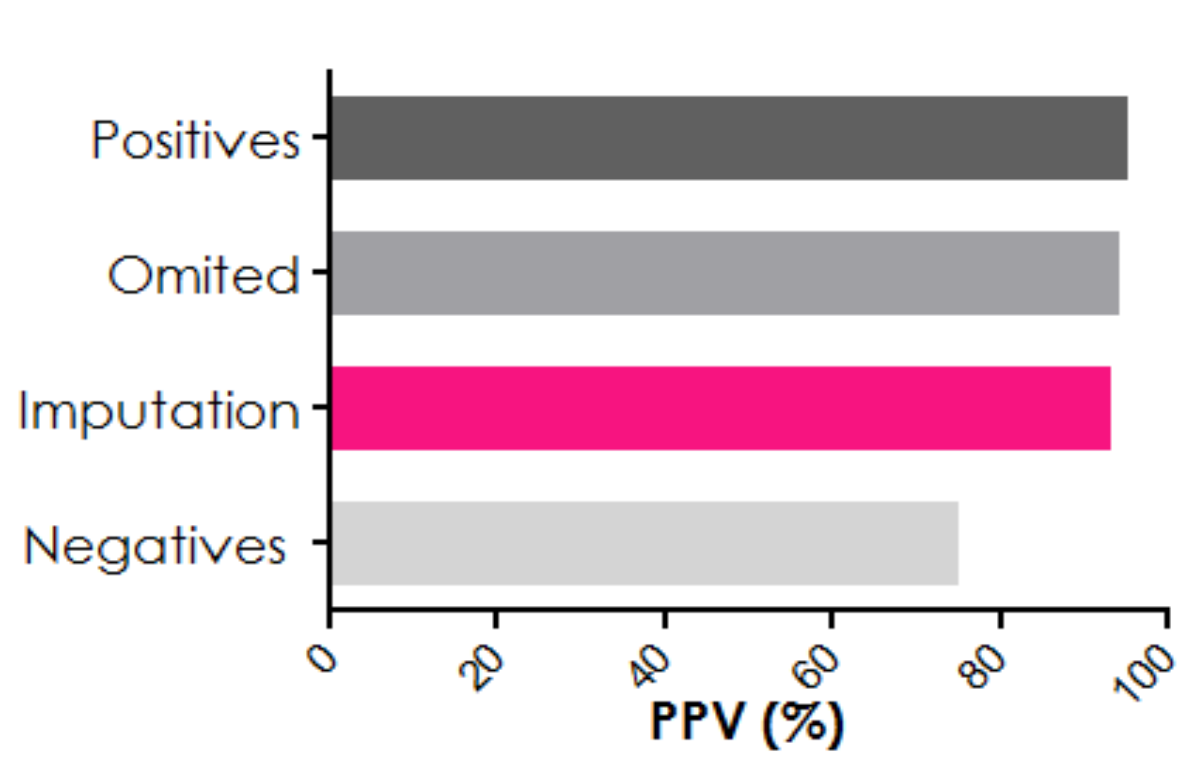

Missing $=$ Negative Results $75.3 \% \mathrm{Cl}(95 \%)(73.9-76.7)$

Missing $=$ Positive Results $95.7 \% \mathrm{Cl}(95 \%)(95-96.3)$

Missing values omitted $94.6 \% \mathrm{Cl}(95 \%)(93.7-95.4)$

\section{Conclusion}

The PPV of bronchiolitis due to RSV was $94 \%$. Only $6 \%$ of the diagnoses would be incorrect. 